\title{
La estructura del diseño curricular
}

\section{The structure of curriculum design}

\section{Referencia del ensayo}

\author{
- Domingo Pérez Brito \\ - Universidad de San Carlos de Guatemala \\ perezbrito42@yahoo.com \\ https://orcid.org/0000-0003-3783-1951
}

Pérez Brito, D. (2020). La estructura del diseño curricular. Revista Guatemalteca de Educación Superior, 3(1), 85-95. DOI: https://doi.org/10.46954/revistages.v1i1.7

Recibido 07/05/2020.

Revisado 08/05/2020.

Aceptado 20/06/2020.

\section{Resumen}

El diseño curricular o currículum, como un edificio, debe contener determinada estructura que engloba el entorno, el marco teórico, el perfil de egreso, los ejes transversales, el plan de estudios y la red curricular, entre otros. Asimismo, integra aspectos legales, administrativos, metodológicos y de evaluación del aprendizaje. Los elementos fundamentales de esta estructura recaen en el perfil de egreso y el plan de estudios. El perfil de egreso permite definir las características futuras del profesional en proceso de formación. Tradicionalmente, se ha estructurado con el esquema de conocimientos, habilidades y actitudes. No obstante, en la actualidad se ha generalizado el enfoque de competencias impulsado por el Proyecto Tuning América Latina que enfatiza en el desempeño del estudiante en situaciones reales y la combinación estrecha de competencias genéricas y competencias específicas de cada disciplina o profesión. El plan de estudios agrupa fundamentalmente las asignaturas y las prácticas de una carrera universitaria. Este agrupamiento puede 
Facultad de Humanidades

Escuela de Estudios de Postgrado

Palabras clave

currículum, perfil de egreso, plan de estudios, metodología, evaluación.

\section{Keywords}

curriculum, egress profile, curriculum, methodology, evaluation ser lineal, modular o mixto. Puede ser un plan de estudios cerrado o flexible. Los currículos cerrados son mayoría, se orientan hacia la especialización disciplinar aunque su rigidez obstaculiza las reformas y actualizaciones curriculares. Al contrario, los currículos flexibles favorecen la interdisciplinariedad, la innovación y diversificación de carreras.

The curricular design or curriculum, like a building, must contain a certain structure that encompasses the environment, the theoretical framework, the graduation profile, the transversal axes, the curriculum and the curricular network, among others. It also integrates legal, administrative, methodological and learning evaluation aspects. The fundamental elements of this structure falls over the Egress Profile and the Curriculum Plan. The egress profile allows the definition of the future characteristics of the graduate in the educational process. Traditionally, it has been structured with a scheme that includes knowledge, skills and attitudes. However, the proficiency approach promoted by the Tuning Latin America Project has become widespread, emphasizing student performance or proficiency in real-world situations and the close combination of generic and specific knowledges, skills and abilities to each discipline or profession. Curriculum fundamentally all courses and practices of a Higher Education career. This gathering can be linear, modular, or mixed. It can be a closed or flexible curriculum. Closed curriculum are the most common, addressed towards disciplinary specialization although their rigidity hinders reforms and curriculum updates. On the contrary, flexible curricula promote interdisciplinary, innovation and career diversification.

\section{Introducción}

El diseño curricular o currículum es un proyecto educativo que tiene la finalidad de contribuir a la resolución de determinados problemas económicos, políticos y sociales a través de la formación de profesionales. La elaboración de este proyecto educativo pasa por cuatro fases: diagnóstico, diseño, ejecución y evaluación curricular. Cada una de ellas cumple una función específica. Por ejemplo, el proceso inicial de diagnóstico curricular sirve para identificar necesidades y demandas de formación profesional, mientras el diseño es la estructuración y 
definición de la propuesta curricular. El currículum debe contener un marco teórico que oriente el trabajo educativo, metas a alcanzar, contenidos y las actividades que será necesario para lograrlas (Zabalza, 2009).

Una vez elaborado, este proyecto educativo adopta diferentes modalidades o tipos: formal, real y oculto. El primero es el documento oficial que sustenta el trabajo de docentes y estudiantes. El segundoeselquegestionaeldocenteenelproceso de enseñanza-aprendizaje y que transforma constantemente, con sus valores, las circunstancias institucionales, el tiempo, los recursos, etc. El tercero es el que resulta de las relaciones y los valores que se fomentan en la escuela y en el aula entre autoridades, docentes y estudiantes.

El presente documento desglosa los principales apartados del diseño curricular que en la Universidad de San Carlos de Guatemala es determinado por la Guía para la elaboración de propuestas curriculares de las unidades académicas y la Guía para la elaboración de programas de postgrado.

\section{Contenido}

\section{La estructura curricular}

En términos formales, el currículum o proyecto curricular debe iniciar con una parte informativa con los datos de la institución, la carrera, el nivel, la especialidad, el lugar, población objetivo, requisitos de ingreso, garantías de calidad, número y calidad del profesorado, ventajas sobre carreras similares, modalidades de graduación, entre otros. La información nos debe proporcionar una idea general aunque breve de dichos estudios.

Un primer apartado clave es el que vincula la propuesta curricular con el entorno, en el sentido que ninguna carrera funciona en el vacío. Este entorno se puede desarrollar desde aspectos globales, nacionales e institucionales. En los aspectos globales y nacionales se puede incluir aspectos económicos, 
políticos, sociales, tecnológicos y educativos. En el plano institucional se puede hacer referencia a los fines y objetivos de la institución, visión y misión, planes estratégicos, programas, proyectos, financiamiento, recursos materiales y humanos, fortalezas, ventajas, etc. El entorno y el proyecto educativo se influyen mutuamente; mientras el contexto socioeconómico condiciona el modelo educativo, la educación "incide" en el futuro de la sociedad (Casanova, 2009). Para César Coll, el currículum debe integrar tres elementos: las "condiciones" o contexto, los principios generales y la práctica pedagógica (Coll, 2009).

El siguiente apartado fundamental se refiere a las fuentes del currículum. Su selección y definición depende de cada institución educativa; no obstante, una idea generalmente aceptada es que se incluyan, como mínimo, los fundamentos filosóficos, pedagógicos y psicológicos. El primero hace referencia a la sociedad, la cultura y a los saberes. El segundo se centra en las características del modelo educativo, los principios metodológicos del proceso de enseñanza-aprendizaje, la función de la institución, del docente y del estudiante. El tercero se enfoca en explicar las nociones sobre el aprendizaje, la persona y los tipos de personalidad, entre otros. En general, este apartado permitirá delinear el modelo y el perfil educativo de la institución (Casarini, 2007).

Tabla 1. Componentes del diseño curricular

1. Información general

2. Entorno: global, nacional, institucional

3. Fuentes del curriculum

4. Perfil: de ingreso y de egreso

5. Ejes curriculares

6. Objetivos de la carrera

7. Áreas curriculares

8. Plan de estudios y red curricular

9. Orientaciones metodológicas y de evaluación del aprendizaje

10. Órganos e instrumentos reguladores

Fuente: USAC, 2004. Guía para la elaboración de propuestas curriculares. 


\section{El perfil de egreso}

Posteriormente, se procede a la construcción de los perfiles de ingreso y de egreso. El perfil de ingreso define los aspectos personales y educativos que debe poseer el candidato a determinados estudios, aspectos que le facilitaran su desenvolvimiento académico y la culminación exitosa de la carrera que está por iniciar. El perfil de egreso hace referencia a las capacidades y habilidades que el estudiante debe poseer al culminar la carrera, que construirá paulatinamente durante el transcurso de su formación y que la institución se compromete a certificar. Sobre el perfil de egreso, Zabalza indica:

"La definición del perfil posee una especial importancia por cuanto va a actuar como un punto de referencia y de guía de todo el resto del proceso. Los contenidos que se seleccionen, las prácticas que se incorporen, la secuencia en que todo ello se integra va a estar condicionada por el tipo de perfil profesional al que esté vinculado un determinado plan de estudios". (Zabalza, 2011).

Tradicionalmente, el perfil de egreso se estructura con los apartados de conocimientos, habilidades y actitudes. No obstante, esta modalidad se ha ido abandonando paulatinamente a favor del enfoque por competencias, que es una tendencia dominante no solo en educación superior sino en todos los niveles educativos y también en los programas de formación laboral. De los diversos modelos y propuestas, el modelo de competencias genéricas y competencias específicas impulsado por el Proyecto Tuning América Latina, ha iniciado un lento aunque persistente proceso de implantación en la Universidad de San Carlos de Guatemala. 
Facultad de Humanidades

Escuela de Estudios de Postgrado

Tabla 2. Perfil de egreso

\begin{tabular}{ll}
\hline \multicolumn{1}{c}{ Apartado } & \multicolumn{1}{c}{ Contenidos } \\
\hline Declaración general & Propósitos generales de la carrera. \\
\hline Ámbitos de acción & Los dominios, campos o espacios laborales de la carrera. \\
\hline Competencias generales & $\begin{array}{l}\text { Relacionadas con todas las disciplinas profesionales como } \\
\text { resolución de problemas, comunicación, trabajo en equipo, } \\
\text { etc. }\end{array}$ \\
\hline Competencias específicas & Propias de cada carrera y especialidad. \\
\hline
\end{tabular}

Fuente: Proyecto Tuning América Latina, 2014.

El siguiente paso es el desarrollo de los ejes y las áreas. Los ejes curriculares están relacionados con grandes temas económicos, políticos y sociales que no es posible incluir en el plan de estudios, pero cuyo estudio, por la naturaleza y carácter de la formación profesional, es casi imposible ignorar. Además, tienen estrecha relación con el perfil institucional y el modelo educativo. Por ejemplo, la Universidad de San Carlos de Guatemala considera como ejes de programas y proyectos los temas de ambiente, género e interculturalidad. (USAC, 2003) Asimismo, cada unidad académica define sus propios ejes institucionales y curriculares.

Las áreas del currículo son los grandes bloques de cursos homogéneos dentro del plan de estudios. En la experiencia institucional de la Universidad de San Carlos de Guatemala, los planes de estudio de todas las carreras tienen tres grandes áreas: el área social-humanística, el área científica-tecnológica y el área disciplinaria. La primera comprende los cursos humanísticos y de cultura general. La segunda comprende los cursos de ciencias básicas y la tercera abarca a los cursos propios de la disciplina. $\mathrm{Si}$ se considera conveniente, pueden incluirse subáreas, especialmente en el área disciplinaria o profesional. Por ejemplo, en la licenciatura en Ciencias Jurídicas y Sociales, es posible que exista una subárea de derecho penal, otra subárea de derecho constitucional, entre otras. 


\section{El plan de estudios}

La primera etapa da paso a la construcción del apartado más importante del diseño curricular: el plan de estudios, que Zabalza presenta como:

"La expresión más genuina del currículo formativo universitario está constituida por los Planes de estudio. En ellos se prefigura el sentido y los contenidos de la formación que la institución universitaria pretende ofrecer a los estudiantes". (Zabalza, 2011).

El plan de estudios estructura la carrera por niveles y por ciclos, además de ordenar la secuencia y desarrollo de asignaturas y prácticas a través de tres enfoques principales: de lo general a lo específico, de lo particular a lo general y por proyectos. (Posner, 2005) Las asignaturas contemplan los saberes conceptuales, procedimentales y actitudinales. Las prácticas se distribuyen o separan en prácticas de curso, trabajos de campo, experiencias con la comunidad, pasantías hasta terminar con el Ejercicio Profesional Supervisado, EPS. Un tema que se debate constantemente consiste en el dilema de cómo incorporar prácticas en todo el recorrido curricular y no solo al final. Para Mateos y Montanero:

"El plan de estudios debe recoger los objetivos, materias y orientaciones metodológicas respecto de la docencia y a la evaluación; del título, así como otros aspectos relativos a los recursos y agentes implicados como los estudiantes, profesores, personal de administración y servicios". (Mateos y Montanero, 2008)

Asimismo, el plan de estudios puede estructurarse de manera lineal, por módulos o una combinación de ambas. Igualmente puede ser rígido o flexible. En la experiencia acumulada de la USAC, la preeminencia de los planes de estudios rígidos, cerrados y disciplinarios es aplastante; a excepción de la Facultad de Ingeniería. Los planes cerrados se orientan hacia la profundización y a la especialización. Por el contrario, los 
currículos flexibles facilitan los procesos de innovación, permiten la incorporación de cursos optativos o electivos, además de fomentar la diversificación de carreras. Es una tendencia que no ha crecido aparentemente por motivos de costo, no obstante sus ventajas. Para Zabalza, la flexibilidad curricular es impulsada por las demandas del mercado laboral y por la evolución de las profesiones. Señala que los currículos flexibles facilitan la incorporación de niveles, áreas comunes, el fomento de las equivalencias y la incorporación de materias optativas, (Zabalza, 2011).

Tabla 3. Plan de estudios

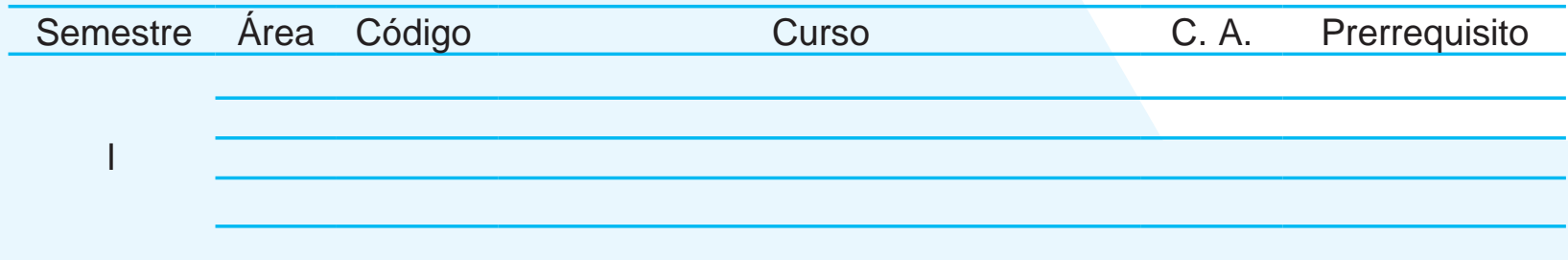

Fuente: USAC, 2004. Guía para la elaboración de propuestas curriculares.

El esquema del plan de estudios debería incluir información sobre las áreas curriculares, códigos de los cursos, las asignaturas y las prácticas, los créditos académicos y la secuencia de los prerrequisitos. Igualmente, se elabora la red curricular, que es la representación gráfica del plan de estudios. Facilita la visualización de la carrera y la evolución por niveles y ciclos.

Tabla 4. Red curricular

\begin{tabular}{c|l|l|l|l|l|l|l|l|l|l}
\hline \multirow{2}{*}{ Áreas } & \multicolumn{7}{|c|}{ Semestres } \\
\hline & & & & & 5 & & & & & \\
\hline \multirow{3}{*}{1} & & & & & & & & & & \\
\cline { 2 - 11 } & & & & & & & & & & \\
\hline \multirow{2}{*}{2} & & & & & & & & & & \\
\cline { 2 - 11 } & & & & & & & & & & \\
\hline \multirow{2}{*}{3} & & & & & & & & & & \\
\cline { 2 - 11 } & & & & & & & & & & \\
\hline
\end{tabular}

Fuente: USAC, 2004. Guía para la elaboración de propuestas curriculares. 
Después de la red curricular, es recomendable incluir contenidos mínimos de las asignaturas, prácticas y demás actividades académicas de la carrera. De igual manera, es útil elaborar un apartado institucional sobre las orientaciones metodológicas y los criterios de evaluación.

Como un apartado final, la estructura del diseño curricular hace referencia a los órganos e instrumentos reguladores del currículo. Los órganos son las instancias institucionales encargadas de dar seguimiento, gestionar y actualizar el diseño curricular. En el caso de la Universidad de San Carlos de Guatemala, son las instancias de coordinación académica que trabajan conjuntamente con las comisiones de rediseño curricular; por ejemplo, en la Facultad de Agronomía, la Unidad de Planificación y Desarrollo Educativo de Agronomía, UPDEA; en la Facultad de Arquitectura, la Dirección de Planificación; en la Facultad de Ciencias Químicas y Farmacia, el Centro de Desarrollo Educativo, CEDE.

Los instrumentos reguladores son los reglamentos necesarios para la gestión y la administración del currículum, como los reglamentos de evaluación, de escuela de vacaciones, de graduación, de EPS, etc. Son todos aquellos normativos y reglamentos que permiten dar viabilidad y sostenibilidad al diseño curricular. Es la base legal.

\section{Conclusión}

Una propuesta o proyecto curricular siempre responde a determinadas necesidades y demandas económicas, políticas, sociales y educativas, tomando en consideración que el proceso de diseño curricular transita por las fases de diagnóstico, diseño, ejecución y evaluación curricular. El perfil de egreso y el plan de estudios se convierten en los componentes básicos y fundamentales del diseño curricular. Es importante establecer que la gestión del diseño curricular plantea la existencia de órganos e instrumentos reguladores. 


\section{Referencias}

Casanova, M. A. (2009) Diseño curricular e innovación educativa. Madrid: La Muralla.

Casarini, M. (2007) Teoría y diseño curricular. México: Trillas.

Coll, C. (2009) Psicología y curriculum. Barcelona: Paidós.

Mateos, V. L. y M. Montanero. (Coords.) (2008) Diseño e implantación de títulos de grado en el espacio europeo de educación superior. Madrid: Narcea.

Posner, G. (2005) Análisis del currículo. México: McGraw-Hill.

Proyecto Tuning América Latina. (2014) Meta perfiles y perfiles. Una nueva aproximación para las titulaciones en América Latina. Bilbao: Universidad de Deusto. Editores: P. Beneitone, J. González y R. Wageenaar.

USAC. (2003) Plan estratégico USAC 2022. Resumen ejecutivo.

USAC. (2004) Guía para la elaboración de propuestas curriculares de las unidades académicas de la Universidad de San Carlos de Guatemala.

USAC. (2015) Guía para la elaboración de programas de postgrado. Sistema de Estudios de Postgrado.

Zabalza, M. A. (2009) Diseño y desarrollo curricular. Madrid: Narcea.

Zabalza, M. A. (2011) Competencias docentes del profesorado universitario. Calidad y desarrollo profesional. Madrid: Narcea. 


\section{Sobre el autor}

Domingo Pérez Brito es Licenciado en Letras por la Universidad de San Carlos de Guatemala y Master en Currículo por la Universidad del Valle de Guatemala. Ha trabajado en el sector público, privado y no gubernamental. Ha escrito sobre educación, currículo y literatura. Actualmente trabaja como profesional de desarrollo curricular en el Departamento de Asesoría y Orientación Curricular.

\section{Copyright (c) Domingo Pérez Brito}

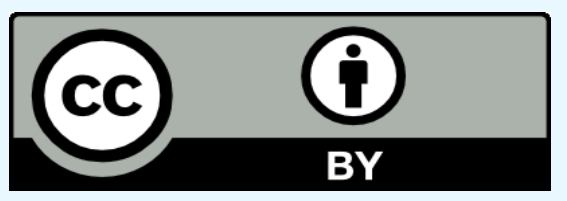

Este texto está protegido por una licencia CreativeCommons 4.0.

Usted es libre para compartir, copiar y redistribuir el material en cualquier medio o formato y adaptar el documento, remezclar, transformar y crear a partir del material para cualquier propósito, incluso comercialmente, siempre que cumpla la condición de atribución: usted debe reconocer el crédito de una obra de manera adecuada, proporcionar un enlace a la licencia, e indicar si se han realizado cambios. Puede hacerlo en cualquier forma razonable, pero no de forma tal que sugiera que tiene el apoyo del licenciante o lo recibe por el uso que hace. 